\title{
A Xon Signature in the Electron Spin
}

\author{
Jean-Paul Auffray \\ (ex) Courant Institute of Mathematical Sciences, New York University, New York, USA \\ Email:.jpauffray@yahoo.fr
}

Received August 25, 2013; revised September 26, 2013; accepted October 25, 2013

Copyright (C) 2013 Jean-Paul Auffray. This is an open access article distributed under the Creative Commons Attribution License, which permits unrestricted use, distribution, and reproduction in any medium, provided the original work is properly cited.

\begin{abstract}
The elementary particles listed in the Standard Model of particle physics have all in common a quantum mechanical attribute which has the dimension of the xon, suggesting that the xon might be a structural ingredient of matter. The xon should therefore be included as a full-fledged member in the SM catalog of elementary particles.
\end{abstract}

Keywords: Observable Universe; Dark Matter; Standard Model; Ordinary Matter; Electron Spin; Xon; Photon; Gravitomagnetism; Smolin; Haroche; Arbab

\section{Introduction}

On March 21, 2013, the Planck mission team working under the auspices of the European Space Agency (ESA) released data suggesting that the total mass-energy present in the observable universe consists in $68.3 \%$ dark energy, $26.8 \%$ dark matter and only some $4.9 \%$ "ordinary" matter [1]. If this is true, then one might be tempted to consider ordinary matter to constitute some kind of "oddity" in the universe.

Is this really the case?

In a preceding communication, we suggested that the xon might play a structural role in the occurrence of dark matter in the universe [2]. In the present note, we examine the possibility that it might also be a structural ingredient of ordinary matter.

\section{Reviewing the Structural Ingredients of Ordinary Matter}

According to the (current) estimates proposed in its prospective catalog by the Standard Model of particle physics (SM), ordinary matter is thought to be composed out of sixty-one different particles, all said to be "elementary" (i.e. they cannot be subdivided into parts smaller than themselves) arranged in a system of eleven "generations" [3].

Second and third generations charged particles decay with very short half-lives and are observed only in highenergy environments.

Among the first generation charged particles that do not decay, together with the proton (thought to be made up of confined quarks), the electron stands as the major constitutive ingredient of matter as we can see it commonly here on Earth-it is not dark.

\section{The Heart of the Mystery}

Each of the particles listed in the SM catalog is said to be dotted with certain intrinsic attributes, such as a restmass or a charge for some of them. Remarkably, with the noteworthy exception of the (hypothetical) Higgs boson not yet observed, ONE - and only one-of these attributes is common to all the particles belonging in the SM system. This odd fact would seem to indicate that this particular attribute might play a special role in determining the structure of the elementary particles constitutive of ordinary matter.

Let us examine what is involved.

\section{Visiting the Electron}

In May 1925, attempting to understand the workings of the Exclusion Principle that Wolfgang Pauli had just formulated, Samuel Goudsmit, then a graduate student at the University of Leiden, formed the idea that the electron perhaps possessed a magnetic moment. When he related his idea to his friend George Uhlenbeck, a graduate student like himself, Uhlenbeck exclaimed: "But don't you see what this implies? It means that there is a fourth degree of freedom for the electron. It means that the electron has a spin-that it rotates [4]."

Thus was born - in two steps and in the reverse order of what is usually explained in physics textbooks - the 
concepts of the electron spin and its associated magnetic moment.

\section{Rethinking the Spin}

In fine, even though the analogy with a classical spinning top or classical rotating sphere was almost at once deemed to be inappropriate, the electron was recognized to be dotted with an attribute having the dimension of an angular momentum (hence the designation "spin" given to it).

Quantum physicists have generally been satisfied with this "definition" ever since. Few if any have paid much attention to another aspect of the invention: angular momentum in general (and therefore the electron spin in particular) has the dimension of...

Dynamical action.

Since action is quantized - composed of action elements, xons in our terminology - then angular momentum, and the electron spin in particular, must also be quantized - composed of (contain) xons in our terminology.

This is of fundamental importance for the purpose of the present Note.

\section{The Electron in a Magnetic Field}

As we reviewed in the preceding Section, connected to its spin the electron possesses a magnetic moment, one being responsible for the other. When placed in a magnetic field (Zeeman effect) the magnetic dipole causes one component (projection) of the spin vector to assume one of two possible allowed orientations with respect to the field axis, conventionally called "up" and "down". Remarkably, the spin component thus affected can be induced to "flip" over (to jump) from one orientation to the other. This occurs when the electron is made to emit (or absorb)... not energy, but one action element-a xon in our terminology.

Let us examine the implications of this quantum mechanical phenomenon.

\section{Lee Smolin's “Mystery”}

In February 1927, then twenty-six years old and working with Nobel Laureate Niels Bohr in Copenhagen, young Werner Heisenberg addressed the vexing problem of finding appropriate new ways to "visualize" concepts and physical processes identified in the framework of the then emerging quantum theory. Rather than attempt to achieve a conceptual "visualization" [Anschauung] of the processes involved in terms of "analogous" classical processes (as had been done at first for the electron spin for instance), Heisenberg constructed the notion of what he called quantum mechanical "intuitive visualizability" [Anschaulichkeit].
To illustrate his purpose, the young inventor formulated what has since been called the "Heisenberg Uncertainty Principle".

According to an example he gave during a conference he held in Chicago three years later, the Principle runs as follows.

Let $\Delta x$ represents the uncertainty in determining the precise position in space of an electron and let $\Delta p$ represents the corresponding uncertainty in determining precisely the momentum (speed) of the same electron. Then, by the Uncertainty Principle, the following quantum mechanical relation applies:

$$
\Delta x \Delta p \geq h .
$$

where $h$ designates "Planck's constant"-i.e. the value of the action content of a xon in our terminology.

Writing three-quarters of a century later, Quantum gravity expert professor Lee Smolin presented this result in those words: "Heisenberg's uncertainty principle asserts that we can only ever measure accurately either the position or the direction and speed of motion of a particle." To which he added this telling comment: "This is part of the mystery - and, to be honest, no one knows how it comes about [5]."

\section{Resolving Lee Smolin’s “Mystery”}

In the framework of the theoretical scheme we have presented in our preceding Note, Equation (1) does speak for itself: it simply states in mathematical language that, having the dimension of dynamical action, Planck's action element $h$ is, as it should, the composition of an extension $-\Delta x$ - and an intensity $-\Delta p$.

This is the very heart of the matter. Lee Smolin's "mystery" is thereby readily dissipated.

As a consequence, incidentally, one might be tempted to suggest that the Uncertainty Principle should (could) be renamed the "Action Principle".

What conclusions can one draw from all of this?

Before we draw conclusions, let us consider one additional quantum mechanical problem which is somewhat vexing.

\section{A Conflict Instituted Right from Day 1}

In 1900, as he related it himself later, Berlin physicist Max Planck uncovered (quasi accidentally) the famous new "universal constant of nature" he designated as $h$ and named the "elementary quantum of action" [Elementares Wirkungquantum] because, he explained, "it had the dimension of action (energy $\times$ time) [6]."

Max Planck then sought to express his discovery in terms more familiar to physicists at large (and to himself!) than those arising from the subtle and somewhat abstract concept of dynamical action that Leibniz had forged 
during his historic trip to Rome to visit the pope in the interest of peace in Europe that was threatened by French king Louis XIV's territorial ambitions (the concept remained totally foreign to Isaac Newton, for example). To that effect, Planck turned his attention to the equation known today as the "Planck relation" which expresses the amount of energy E a heated (black) body radiates at the frequency $v$. He wrote it as:

$$
E=h v \text {. }
$$

which was not necessarily the best choice as we shall see in a moment. He then interpreted it as indicating the presence in the radiated light of "energy elements" $\varepsilon$, soon to be renamed (by Einstein) "light quanta", then photons, designation coined in 1926 (with a different meaning!) by Massachussetts chemist Gilbert N. Lewis from the Greek $\varphi \tilde{\omega} \varsigma, \varphi \omega \tau o ́ \varsigma$, light.

The name has remained. Unlike the action element, however, Planck's $\varepsilon$ cannot properly be said to constitute "elements" in the strict sense of the word inasmuch as the energy they are supposed to carry can assume any value, from 0 to $\infty$, not just one "elementary value", the same for all these "elements" (as is the case with the value $h$ for the action elements). In brief, playing on words, Planck's $\varepsilon$ may be called energy elements, or energy quanta, or photons, but, unlike the action elements, they cannot properly be said to constitute elementary quanta.

\section{The Photon, Reality or... Fiction}

The "energy quantum" or photon concept was invented in the framework of a tense debate that opposed two Giants of theoretical physics at the time, L. Boltzmann and Baltic German chemist Friedrich Wilhelm Ostwald (who was to receive the Nobel prize for chemistry in 1909).

Against Boltzmann, father of the Kinetic Theory of Gases, Ostwald held for what he called Anthropic Physics (physics pertaining to humanity) placing Rankine's energy concept at the heart of all human activities and of physics in particular under the designation Energetics.

Living with his family in Milan in 1901 and unemployed, twenty-two years old Albert Einstein was feverishly in search of a job. Seeing his distress, his father Herman Einstein wrote to Ostwald, then a professor at the University of Leipzig, just about begging him to enroll his son as one of his assistants: "It is you, highly honored Herr Professor, he told Ostwald, whom my son seems to admire and esteem more than any other scholar currently active in physics [7]."

Four years later, perhaps still under the influence of Ostwald's ideas concerning energy, young Einstein intervened in the raging debate concerning the emerging quantum theory. From Berne in 1905, three days after celebrating his twenty-six birthday, he sent to the review
Annalen der Physik his famous "Heuristic" paper which contains this telling statement: "Monochromatic radiation of low intensity [...] behaves thermodynamically as if it consisted of mutually independent energy quanta [lichtquanta] of magnitude $R \beta v / N$ [8]."

In this expression, for some reason unknown to us, Einstein chose to represent Planck's constant as $\beta$ instead of the usual $h$. As for the other symbols which appear in the expression he said: " $R$ denotes the universal gas constant, $N$ the number of 'real molecules' in one gramequivalent" and $v$ the frequency of the monochromatic radiation.

Thus was born officially the concept of light quanta [lichtquanta], photons for us today.

Behaves "as if"... wrote Einstein. He was wise not to have asserted that this appearance necessarily describes a reality.

A numerical example will help uncover the reality beyond the appearance.

\section{The Reality beyond the Appearance}

Suppose the monochromatic radiation Einstein considered had the frequency

$$
v=1000 \mathrm{~s}^{-1} .
$$

this particular value being chosen for the sole purpose of ease of presentation.

Suppose further that Max Planck had written his energy relation as he might (should) have, in the form

$$
E=v h
$$

with the factor $v$ placed in front of (instead of behind) the factor $h$. Then, substituting the value (2b) for $v$ in Equation $(2 \mathrm{c})$, this equation reads

$$
E=1000 \mathrm{~s}^{-1} \cdot h
$$

and it tells a story quite different from what Planck and young Einstein after him had imagined: it describes the radiating body considered as emitting action elementsxons, in our terminology - not photons, at the rate of $1000 h$ per second (or 1 xon with a duration of $1000^{-1} \mathrm{~s}$ ).

The statement is clear: the elements radiated are xons, not Planck-Einstein energy quanta.

By the way, this as if has an illustrious precedent.

In 1932 Russian physicist Igor Yevgenyevich Tamm was confronted with a problem not unlike the problem considered here: how best to describe collective excitation modes in periodic elastic arrangements of atoms or molecules in condensed matter. To solve this problem, Professor Tamm introduced the (mathematical) concept of the phonon. An admitted "fictitious" or "quasi" particle, the phonon nevertheless acts as if it was a real particle, physically present in the solids concerned. 
We submit that the same applies to the photon. Like the phonon, the photon ought to be regarded henceforth to be a mathematically useful but nevertheless fictitious entity deserving to be replaced in the Standard Model of elementary particles by the hitherto unrecognized yet authentic elementary particle, the xon.

\section{Reappraising the Haroche 2012 Nobel Winning Probes}

Let us succinctly review this proposal in the light of significant contemporary events.

Until recently, no one had ever claimed to have isolated a photon long enough to "observe" it. The situation changed dramatically last year when French physicist Serge Haroche was attributed (jointly) the 2012 Nobel Prize for physics after asserting that he and his team at the Laboratoire Kastler Brossel (LKB) in Paris had successfully isolated and "detected" a photon in a specially designed cavity equipped with reflecting mirrors. "This photon, said Dr Haroche, has survived 0.476 s (3.7 cavity lifetimes), corresponding to a propagation of about 143,000 $\mathrm{km}$ between the cavity mirrors [9]."

The mirrors in Dr Haroche's LKB cavity were $1.7 \mathrm{~cm}$ apart. As light quanta, photons are reported to travel permanently at the speed of light. To perform the task described above while travelling at the speed of light299,792,458 $\mathrm{ms}^{-1}$ _-Dr Haroche's captured photon would have had to accomplish over 8 billions uninterrupted trips between the mirrors, bouncing off their surfaces not randomly but at a perfect perpendicular angle (otherwise it would have swiftly decayed) — all of that without losing an iota of its energy and without causing any "damage" to the mirrors which caused it to bounce. This extraordinary performance would have greatly surprised another famous Nobel laureate for physics, the memorable Richard Feynman, who, not long ago - in Los Angeles in 1983 - described unambiguously to a captivated audience what happens when a photon hits the reflecting surface of a glass mirror. "Light is not really affected by surfaces, he said. An incoming photon is scattered by the electrons in the atoms inside the glass, and a new photon [Richard Feynman's emphasis] comes back up [10]."

If Quantum Physics is to retain its legitimate integrity, then the Haroche/Feynman opposite views concerning the photon's ability to reflect off the surface of mirrors must be reconciled.

\section{Out of the Magician Hat}

To achieve this objective, let us consider this question: where does the energy a photon is supposed to carry come from?

It cannot arise from the famed relation $E=m c^{2}$ since photons have zero rest-mass.
On the other hand, as remarked above, photons are reported to travel permanently at the speed of light, $c$. Therefore they carry a linear momentum $p$ such that

$$
E=c p
$$

The energy they carry arises from this relation.

Or does it?

Let us analyse this relation in terms of dimensions, always a tale-telling, easily applied recipe.

Inasmuch as it measures a velocity, the factor $c$ in Equation (3a) has the dimension $l / d$, with $l$ the distance covered in a time of duration $d$. Equation (3a) then reads

$$
E=(l / d) \cdot p
$$

which yields

$$
E d=l p
$$

And here comes out of the Magician Hat... not the hoped-for photon, but... Planck's old elementary quantum of action $h$ once again: $E d=l p=h$-the xon!

The Haroche/Feynman dilemma is resolved if one assumes the photon to be, like the phonon, a useful but nevertheless fictitious entity, yielding an attractive new Quantum Physics in the framework of which the spectacular LKB Haroche photon probes can assume their full value under a new light: the entity Dr Haroche and his LKB team trapped and detected in their cavity was not a photon-more brilliantly it was... a xon!

\section{Searching for Gravitomagnetism}

In the framework of his pioneering efforts to generalize Newton's Law of Gravitation, Arbab Ibrahim Arbab has recently investigated the possible existence in nature of gravitomagnetism. He asked: "The question is what is the gravitomagnetic field? [11]."

It would seem to us that one cannot exclude a priori the possibility that the question thus formulated might be related, at least to some degree, to the theoretical analysis concerning spin and its associated magnetic moment we have developed in the present Note.

On the basis of these considerations we are now ready to draw appropriate conclusions.

\section{Conclusion}

With the noteworthy exception of the (hypothetical) Higgs boson, not yet observed, the elementary particles listed in the Standard Model catalog of elementary particles have all in common a single attribute, spin. Spin has the dimension of dynamical action. Action occurs in nature in the form of elements - xons in our terminologyall containing the same quantity (quantum) of action. The xon thus appears to have impressed its signature in the intrinsic spin all SM particles possess, hence to be a fun- 
damental constitutive ingredient of ordinary matter thereby deserving to be included in the SM catalog as an elementary particle in its own rights - replacing the photon? If, as we suggested in our preceding Note, the xon is also involved in the occurrence of dark matter in the universe, then a subtle hitherto unnoticed potential connection may exist between dark and ordinary matter.

\section{Acknowledgements}

We wish to express our gratitude to the editors and staff of the Journal of Modern Physics for their valuable help in properly preparing this Note for publication.

\section{REFERENCES}

[1] Planck Collaboration, "Planck Collaboration," 2013. http://arxiv.org/pdf/1303.5062v1.pdf

[2] J.-P. Auffray, Journal of Modern Physics, Vol. 4, 2013, pp. 121-125. http://dx.doi.org/10.4236/jmp.2013.48A011

[3] S. Braibant, G. Giacomelli and M. Spurio, "Particles and Fundamental Interactions. An Introduction in Particle Physics," Springer, New York, 2009, pp. 313-314.
[4] J. H. van der Waals, "Foundations of Modern EPR," World Scientific, Singapore, 1998.

[5] L. Smolin, "Three Roads to Quantum Gravity," Weldenfeld and Nicolson, London, 2000, p. 36.

[6] M. Planck, "Scientific Autobiography," 1950, p. 43. http://fr.scribd.com/doc/41698869/Max-Planck-Planck

[7] H. Einstein, "The Collected Papers of Albert Einstein," Vol. 1, Princeton University Press, Princeton, 1987.

[8] A. Einstein, "The Collected Papers of Albert Einstein," Vol. 2, Princeton University Press, Princeton, 1987.

[9] S. Gleyzes, S. Kuhr, C. Guerlin, J. Bernu, S. Deléglise, U. B. Hoff, M. Brune, J.-M. Raimond and S. Haroche, "Quantum Jumps of Light Recording the Birth and Death of a Photon in a Cavity," Nature, Vol. 446, 2007.

[10] R. P. Feynman, "QED, The Strange Theory of Light and Matter," Princeton University Press, Princeton, 1985, pp. 101-104.

[11] A. I. Arbab, Journal of Modern Physics, Vol. 3, 2012, pp. 1231-1235. http://dx.doi.org/10.4236/jmp.2012.329159 